\title{
Other Types of Training
}

National Cancer Institute

\section{Source}

National Cancer Institute. Other Types of Training. NCI Thesaurus. Code C18918.

Other types of training not listed 